\title{
EFFICIENT MANET- INTERNET INTEGRATION FOR MOBILE DEVICES
}

MAHESH KUMAR TIWARI ${ }^{1}$, UDAI SHANKAR ${ }^{2}$, AJAY KUMAR ${ }^{3}$

123 Department of Computer science \& Engineering and Information Technology

${ }^{123}$ Uttar Pradesh Technical university, Uttar Pradesh, India

\section{Maheshyogi26@gmail.com,udaishankar.royal18@gmail.com,ajay_diht@yahoo.co.in} ABSTRACT

A mobile ad hoc network (MANET) consists of wireless mobile nodes without having a fixed infrastructure support. The communication between these mobile nodes is carried out without any centralized control. The communication among the nodes within the infrastructure less networks is done through Ad-Hoc routing protocols, But whenever any mobile node want to communicate to a node in a network that is outside the ad hoc network such as internet, there should be an appropriate mechanism for establishing this connection with the internet node or host. Gateway discovery is a fundamental process in connecting MANET with the internet. A mobile node can connect to the internet by discovery of some specialized nodes called as gateway nodes. These Gateway nodes act as a bridge between the mobile ad hoc networks node and the internet. The basic aim of the gateway discovery approach is to modify the route discovery process so that it is not only used for discovery of destination mobile nodes but also the gateways. In this paper we are going to present a review of various gateway discovery approaches which are used for establishing the interconnection of mobile ad hoc networks and internet and going to propose a algorithm that reduce the discovery time of the gateway by selective forwarding and simultaneously also optimize the gateway discovery control overheads.

\section{Keywords}

Mobile IP; DAD; Address Auto-configuration; Gateway Forwarding; Handoff; Gateway Discovery

\section{Council for Innovative Research}

Peer Review Research Publishing System

\section{Journal: INTERNATIONAL JOURNAL OF COMPUTERS \& TECHNOLOGY}

Vol. 14, No. 1

www.ijctonline.com , editorijctonline@gmail.com 


\section{INTRODUCTION}

A mobile ad hoc network [1] is organized as the collection of various mobile nodes. It is formed spontaneously in the areas where establishing an infrastructure network causes delay in the work as well as enhances the cost. These areas include flooded areas, earthquake affected areas, and other rescue operations etc. As the nodes are mobile there is not any fixed infrastructure of MANET. The mobile ad hoc network is composed of portable nodes, notebooks and palmtops. This portability brings a significant issue of mobility. This is a key challenge in ad-hoc networks. The nodes in the mobile ad hoc networks are constrained to use of limited resources. They have low battery power, limited user interface, low storage memory and low quality display. The nodes in the mobile ad hoc networks can communicate with one-another through the use of some routing protocols which decides which way to route the information from source to destination. This communication among the mobile nodes occurs for retrieving the essential information they need. When a mobile node wants any information outside the mobile ad hoc network in which they are present, they have to use some mechanism other than routing protocol. The communication between mobile ad hoc networks and the internet occurs through some special approach called as gateway discovery and selection. Mobile devices can construct an IP (Internet Protocol) address valid in the domain where they reside. According to the specifications where RA (Router Advertisement) messages are defined [1], these messages cannot be propagated [2]. If this technology is applied as itself in a multi hop wireless networks, those nodes that are outside the coverage area of the Access Router will not be able to obtain a valid IP address and, in turn, they will not be able to access the Internet. Therefore, interworking between conventional protocols and ad hoc network technologies must be studied. In this sense, the current solutions propose the inclusion of an Internet Gateway which is connected to the Access Router and complements it [3]. The Gateway carries out two main tasks. Firstly, it provides the ad hoc routing capabilities that are absent in conventional Access Routers so that the downlink traffic can be conveniently re-routed in the MANET. Secondly, the Gateway broadcasts Modified Router Advertisement (MRA) messages.

The first level of classification (reactive, hybrid, proactive) is based on the TTL (Time-to-live) of MRA messages. The emission of MRA messages may be accomplished on demand from the mobile nodes (reactive), periodically activated by the Gateway (proactive) or combining the previous approaches (hybrid). In the hybrid scheme, MRA messages are periodically broadcast in an area close to the Internet Gateway while the devices located outside this zone demand the MRA information by generating Modified Router Solicitation (MRS) messages [4]. The proactive zone is defined by the TTL value in the MRA messages, that is, the number of retransmissions that an MRA message can have. By adjusting the TTL value, the hybrid gateway discovery can behave as proactive (the TTL set to the network diameter) or as reactive (the TTL set to 0). We can divide the proactive schemes in fixed and adaptive approaches. In the fixed algorithms the interval of emission of MRA messages (T) does not change while in adaptive schemes this value is adapted dynamically as a function of some characteristic of network. The hybrid schemes can also be classified according to the decision on the retransmissions of the MRA messages. When the decision to broadcast the MRA does exclusively depend on the TTL value set by the GW, the algorithm is centralized.
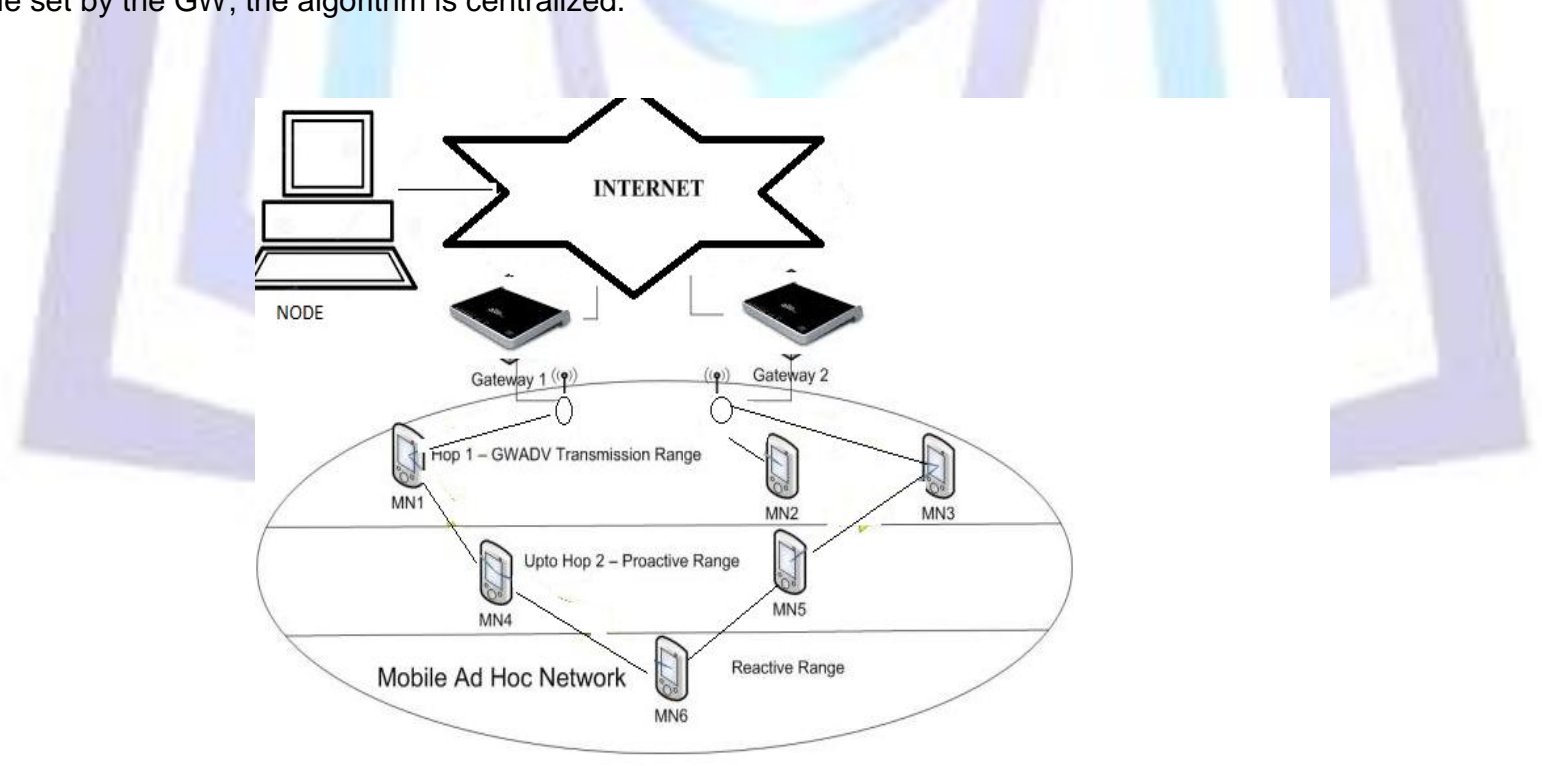

Figure 1. Ad-hoc network model

\section{RELATED WORK}

In [5] the interval (T) and the Time to Live (TTL) should be adapted to the network conditions. One of the first adaptive and centralized algorithms was the Maximal Source Coverage (MSC). In this proposal, the $T$ is set to a fixed value while the gateway will send out the next advertisement message with the TTL equal to the minimum number of hops required to reach all the sources that use this gateway to communicate with external hosts.

In [8] the Adaptive Distributed gateway Discovery (ADD) is presented. In this work, there is a new distributed strategy: only the mobile nodes that are relaying data packets are able to forward the MRA messages. Our proposal also adjusts the 
TTL value of the MRA to the network conditions. To do so, we incorporate a policy in the mobile nodes so that they decide about the retransmission of the MRA messages. This algorithm is explained in the next Section.

In the proposal of Lee et al. [12] a hybrid gateway discovery scheme is presented and it is compared with a reactive approach handling the routing within the MANET it uses a source routing protocol. This limits the applicability and scalability of their scheme. In this approach only upon the detection of the topology change in MANET.

Bin et al. [24], proposed an adaptive gateway discovery scheme that can dynamically adjust the TTL value of Agent Advertisements (GWADV messages) according to the mobile nodes MANET Internet traffic and their related position from Internet Gateways with which they registered. This protocol provides Internet access to MANET mobile nodes using mobile IP.

Hamidian [25], the authors extended the AODV routing protocol to the Internet to achieve the interconnection between the MANET and the Internet. The paper discusses and evaluates three approaches for gateway discovery. The authors implement these three schemes in network simulator 2 (ns-2) and compare them by means of simulation study. They also discuss the advantages and disadvantages of the three approaches. The gateway discovery method follows a similar approach based on. However, those approaches have a fixed TTL for proactive gateway advertisements and do not reflect dynamic network conditions.

The optimization of the timing of MRA messages was studied in R. Kumar [26]. In this study, the authors suggest that the appropriateness of broadcasting a MRA message depends on the number of active sources that communicate to external hosts as well as the number of intermediate nodes that forward the packets to the internet gateway. With these two parameters, the so-called Regulated Mobility Degree (RMD) is defined. When this factor overpasses a pre-established threshold, the MRA message is sent.

Trujillo [27] proposed process optimized adaptive gateway discovery approach to provide internet connectivity in multi-hop ad hoc networks. Modified router advertisement (MRA) message scheme is used to discover the gateway. Claims that mechanism can be implemented using either scheme, reactive, proactive or hybrid. Time interval T is defined for MRA advertisement as beacon interval. Control system function is defined to adjust that is proportional to MRA massages received from the gateway neighbors and also real mobile node close to the gateway. It is also defined that the probability $p$ that a node is near a gateway, regardless of the model chosen for mobility can be given by binomial distribution.

\section{Problem Domain}

Currently there is a plethora of proposals to solve the problems of MANET-INTERNET integration. Many of the designs suffer from complexity and design solutions that have not been properly evaluated. The main issue in MANET-INTERNET integration is the design of internet connectivity for MANETs that can handle node mobility both at micro and macro level that is within the same MANET and between the MANET and another networks, MANET or other fixed wired or wireless networks respectively, having continuous and uninterrupted internet connections whenever there is at least one potential route to one or more gateways.

Three challenging problems in Mobile Ad-hoc network

i) Determine the location of Node

ii) Discovering valuable gateway

iii) Maintain and stabilize the state of Discovered Gateway.

The Major and significant problems based on above scenario discussed that still require to investigate of an efficient and robust network condition independent solution for MANET-INTERNET integration [16][17] has to be address the following problems:

A. Control Overheads: Overheads introduced due to the Gateway solicitation, Gateway advertisement and mobile IP itself.

B. Load balancing: Balancing among the different gateways when connected with same/ different nodes.

C. Multi homing: To maintain the consistent connectivity simultaneously with multiple gateways.

D. Hand Offs: Switching from one gateway to another when the mobile node roams outside the vicinity of the current gateway i.e. to provide smooth seamless roaming.

E. Gateway Selection: To select the optimal gateway from the multiple available gateways.

\section{GateWAy Discovery TeChNiques:}

For access to global services, an Internet Gateway (IGW) in the access network can provide Internet connectivity for nodes in the MANETs. Mobile nodes from Ad Hoc network can use this route to send/receive packets addressed to or from Internet. The standard Ad Hoc routing protocols do not provide the functionality of detecting Internet gateways, thus the protocols have to be extended. The extensions to the standard Ad Hoc routing protocols are based upon special Ad Hoc routing messages. Depending on who initiates the gateway discovery, these approaches can broadly be classified into the following three categories [18, 19, 20].
A. Reactive gateway discovery.
B. Proactive gateway discovery. 


\section{Hybrid gateway discovery \\ D. Adaptive Gateway Discovery}

Ad-Hoc on Demand Distance Vector Routing (AODV) protocol has been studied to provide the connectivity to the wired network (internet and other resources) via gateway. (A modification in the source code of AODV in NS 2 in accordance with the Internet draft "Global connectivity for IPv6 Mobile Ad-Hoc Networks" which presents a solution where AODV is used to provide Internet access to mobile nodes [21]. To always keep the mobile nodes updated about the information of the gateways three mechanisms have been proposed to transmit the information about the gateways to the mobile nodes. The configuration phase between the Fixed network (Gateway) and mobile nodes in the mobile Ad-Hoc network can be initiated by the Fixed network(Gateway), by the mobile node or a mixed approach can be used to configure the mobile nodes and the fixed network. These gateways discovery mechanism are discussed as below:

\section{A. Reactive Gateway Discovery}

The reactive gateway discovery is initiated by a mobile node that is to initialize or update information about the gateway. The mobile node broadcasts a RREQ_I to the ALL_MANET_GW_MULTICAST address [9], i.e. the IP address for the group of all gateways in a mobile Ad-Hoc network. Thus, only the gateways are addressed by this message and only they process it. Intermediate mobile nodes that receive the message just forward it by broadcasting it again. Since the message format is RREQ, which has a RREQ ID field. A, duplicated RREQ_Is are discarded. Upon receipt of a RREQ_I, a gateway unicasts back a RREP_I which, among other things, contains the IP address of the gateway. The advantage of this approach is that RREQ_Is are sent only when a mobile node needs the information about reachable gateways. Hence, the periodic flooding of the complete mobile Ad-Hoc network, that has obvious disadvantages. The disadvantage of reactive gateway discovery is that the load on forwarding mobile nodes, especially on those close to a gateway, is increased.

\section{B. Proactive Gateway Discovery}

The proactive gateway discovery is initiated by the gateway itself. The gateway periodically broadcasts a gateway advertisement (GWADV) message which is transmitted after expiration of the gateway's timer ADVERTISEMENT_INTERVAL. The time between two consecutive advertisements must be chosen with care so that the network is not flooded unnecessarily. All mobile nodes residing in the gateway's transmission range receive the advertisement. Upon receipt of the advertisement, the mobile nodes that do not have a route to the gateway create a route entry for it in their routing tables. Mobile nodes that already have a route to the gateway update their route entry for the gateway. Next, the advertisement is forwarded by the mobile nodes to other mobile nodes residing in their transmission range. To assure that all mobile nodes within the mobile Ad-Hoc network receive the advertisement, the number of retransmissions is determined by NET_DIAMETER defined by AODV. However, this will lead to enormously many unnecessary duplicated advertisements. A conceivable solution to the problem that occurs due to these duplicated advertisements. Although the problem of duplicated broadcast messages can be solved, one disadvantage remains. This disadvantage, which is general for all proactive approaches, is the fact that the message is flooded through the whole mobile Ad-Hoc network periodically. This is very costly operation. Limited resources in a mobile Ad-Hoc network, such as power and bandwidth, will be used a lot.

\section{Hybrid Gateway Discovery}

In the hybrid gateway discovery approach the gateway periodically broadcasts the GWADV message. The TTL is set to ADVERTISEMENT_ZONE so that the advertisement message can be forwarded only up to this maximal number of hops through the ad hoc network. The mobile nodes within this region receive this message and act according to the proactive approach. The nodes outside this region discover the default routes to the gateways using the reactive approach.

\section{Adaptive Gateway Discovery}

To offset changing ad hoc network conditions like node density, transmission quality etc; a static TTL value cannot be used. A modified Hybrid Gateway Discovery mechanism which dynamically adjusts value of TTL and periodicity of GW_ADV messages depending on the MANET characteristics in order to achieve a good trade-off between performance and network overhead is called an Adaptive Gateway Discovery Mechanism [6][14]. Several novel strategies have been proposed recently which implement adaptive gateway discovery mechanisms in different ways.

\section{SELECTIVE FORWARDING}

In the proposed algorithm, the gateway also sends MRA messages every $T$ seconds as conventional hybrid gateway discoveries. However, in this scheme the mobile nodes decide whether to forward them or not according to the residual lifetime of the route to the Gateway. Computing the exact residual lifetime of any route is not a trivial task [9], so in this paper we propose the use of two parameters to estimate the route residual lifetime. These parameters, which are conventionally kept in the route caches, are:

A- Number of hops to the Gateway. Route lifetimes strongly depend on the number of hops that it contains [10] In this sense, a route composed of few nodes is expected to endure longer that a path with more hops. Then, the characteristics of interconnection to the Internet are clearly affected by distance to GW. This fact is shown in Figure 2, which represents the cumulative distribution function of the route lifetime obtained with different scenarios, nodes and speeds. As shown in Figure 2, when the number of hops to the gateway increases, the mean route time decreases. 
B- Route Expiration Time. In reactive ad hoc routing protocols, learnt routes are stored in caches during an interval along which the route is considered to be valid. When this interval is over, if the route has not been employed, the entry is removed from the cache. On the other hand, every time the route is used without any transmission error, its expiration time is updated. Specifically, in AODV (Ad Hoc on Demand Distance Vector), route expiration is increased in ActiveRouteTimeout seconds when it is used [12]. Roughly, our algorithm assumes that those routes whose expiration time is far to expire do not need to be frequently updated because they have been recently discovered or recently used without any transmission error.

C- Relaying for some other nodes. With this variable, we measure if the node is an active source or if it is relaying for some traffic sources. In this way, the MRA messages will be propagated to the areas where updated routes to the Internet are needed.

\section{PROPOSED WORK}

With these three parameters, our algorithm makes MRA messages propagate exclusively in those areas where active sources with unstable routes exit. Additionally, as any node in the MANET may unexpectedly start a communication with an external host, the Gateway periodically generates an MRA message which is mandatorily propagated in the whole MANET. To differentiate these two operations, we have added a new flag to the MRA message. The flag has been called Selective Forwarding (SF).MRA message is forwarded by mobile nodes with active routes as long as the lifetime is about to end. Mathematically, this condition is expressed in the Equation

\section{Expiration lifetime of a particular node $=T^{\star}$ hop}

Where $T$ is the interval of emission of MRA messages, while hop is the number of hops that the route to the Gateway contains. When this condition holds and the node is acting as a relay for other data sources, the MRA message is retransmitted. Independently of the number of hops that they contain, all routes when used or discovered increase their expiration lifetime with constant value

On the other hand, when the SF in the MRA message is cleared, the nodes must retransmit the message. These MRAs, which we will call them mandatory MRAs, are sent in the following two cases:

. The last mandatory MRA was sent $T$ mandatory seconds ago.

The ratio between the number of MRS messages sent by the GW and data sources is higher than $50 \%$. With this condition, the Gateway can detect the situations with a high mobility where it is more convenient to update all the routes to the Gateway proactively.

The algorithm of retransmitting the MRA messages in the mobile nodes is:-

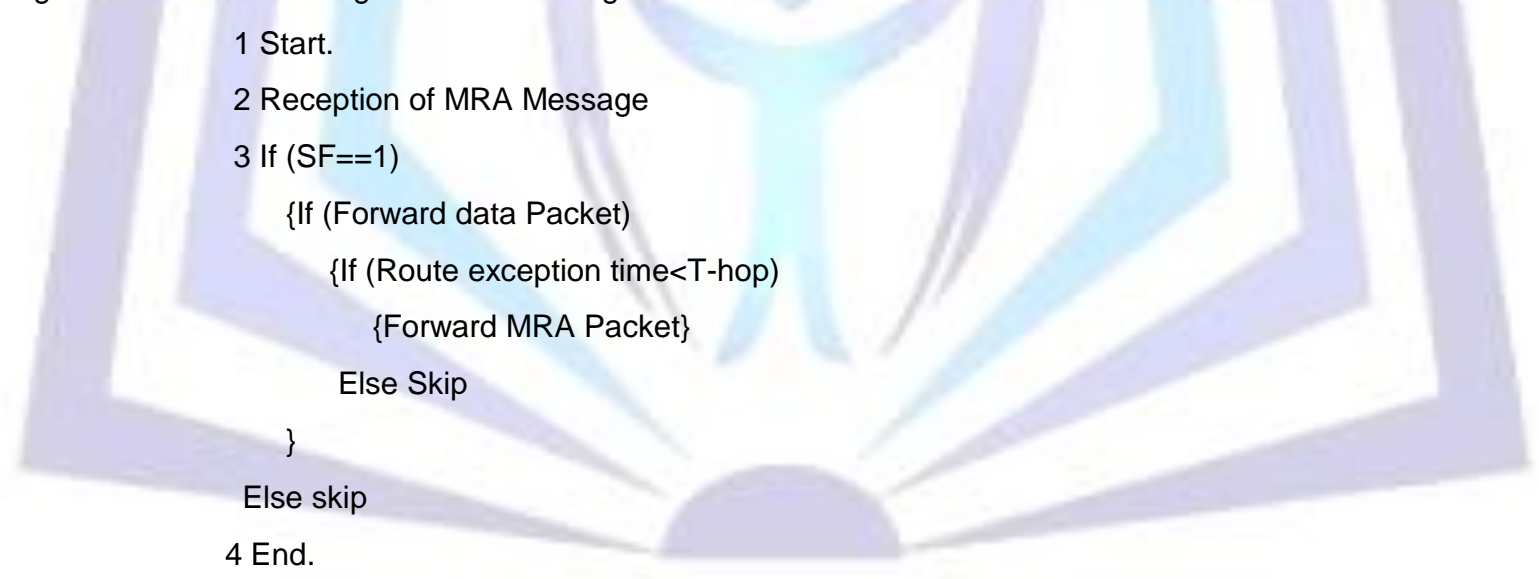

\section{PERFORMANTION METRICS}

Due to the difficulties associated to real tests, the benefits of our proposal for distributed gateway discovery [15] have been verified by the use of simulations. It was necessary to develop a software module that includes the algorithm in the Global Connectivity support [11]. This module has been integrated into the open source Network Simulator tool, ns-2.31[7] on a Linux machine [13]. RMD parameters [22] are set according to simulation parameter. 
Table -1 Simulation parameters

\begin{tabular}{|c|c|}
\hline Simulation parameters & Values \\
\hline Simulation Area & $1500 \times 300 \mathrm{~m} 2$ \\
\hline Number of Nodes & 50 \\
\hline Gateway Location & $(750,150) \mathrm{m}$ \\
\hline Transmission Range & $250 \mathrm{~m}$ \\
\hline Simulation Time & $1000 \mathrm{~s}$ \\
\hline Pause Time & $10 \mathrm{Sec}$ \\
\hline Runs per Point & 100 \\
\hline Ad hoc Protocol & AODV \\
\hline No. of Sources & 10 sources \\
\hline Link Layer & $\begin{array}{l}\text { Link layer detection enabled, } 802.11 \\
\text { RTS/CTS enabled }\end{array}$ \\
\hline Mobility pattern & Maximum speed: 2 to $5 \mathrm{~m} / \mathrm{s}$ \\
\hline Bit Rate & CBR(Constant Bit Rate) \\
\hline Packet Length & 128 bytes \\
\hline Model & Random way point \\
\hline Distributed Proposal & T mandatory: $20 \mathrm{~s}$ \\
\hline
\end{tabular}

We have primarily selected the following three parameters in order to study the performance comparison of the three gateway discovery approaches.

Packet delivery fraction: This is defined as the ratio between the number of delivered packets and those generated by the constant bit rate (CBR) traffic sources.

Average end-to-end delay: This is basically defined as the ratio between the summation of the time difference between the packets received time and the packet sent time and the summation of data packets received by all nodes.

Normalized routing load: This is defined as the number of routing packets transmitted per data packet delivered at the destination. Each hop-wise transmission of a routing packet is counted as one transmission.

\section{SIMULATION RESULTS AND ANALYSIS}

In this section we have studied the effect of the three gateway discovery approaches under varying pause time and increasing number of sources, on the performance of the hybrid ad hoc network.

\section{A. Packet Delivery Fraction (PDF) Comparison}

The packet delivery fraction is measured under varying pause time with 10 and 20 numbers of sources. From Figure we see that the proactive approach has better packet delivery performance than the reactive approach. This happens because - due to the periodic update of route information form the gateway, routes form all the nodes to the gateway are always available. As a result majority of the packets are delivered smoothly. In case of reactive approach, a node wishing to send data to the destination needs to find the route to the gateway first. This takes a certain amount of time and no packet can be sent during this period due to the unavailability of routes. Moreover, in case of proactive approach, due to regular exchange of gateway information, routes are always optimized and the nodes have fresher and shorter routes to the destination. This reduces the chances of link breaks and increases the packet delivery ratio. On the other hand in reactive approach, a node continues to use a longer route until it is broken even if an alternate shorter route is available. With longer and older routes, the chances of link breaks and the dropping of packets also increase. This reduces the packet delivery fraction. The packet delivery performance of the hybrid approach falls between that of the proactive and reactive approaches. 


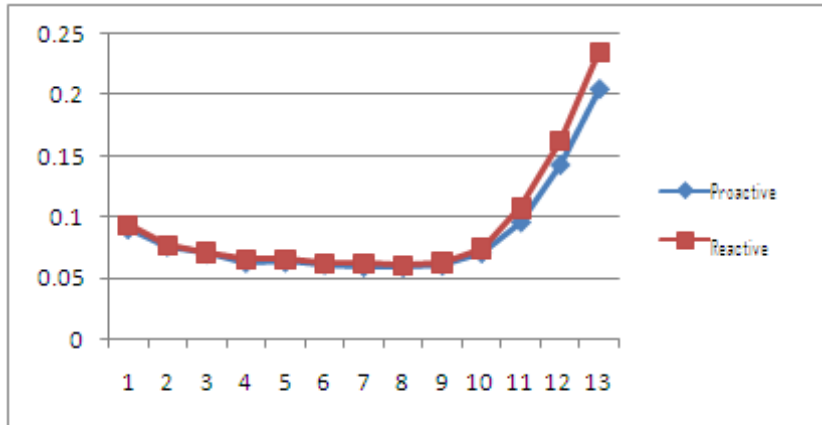

\section{B. Average End-to-End Delay Comparison}

From Figure we see that the average end-to-end delay with the proactive and hybrid gateway discovery approach [23] is less in comparison to the reactive gateway discovery. In proactive approach, due to periodic route updates from the gateway, routes are optimized regularly and the nodes have fresher and shorter routes to the gateway. Moreover, all the routes are maintained all the time. This instant availability of the fresher and shorter routes enables the nodes to deliver packets to their destinations with less delay. In reactive approach, a node needs to find a route to the gateway first before sending the packet. This initial path setup delays the delivery of the packets.

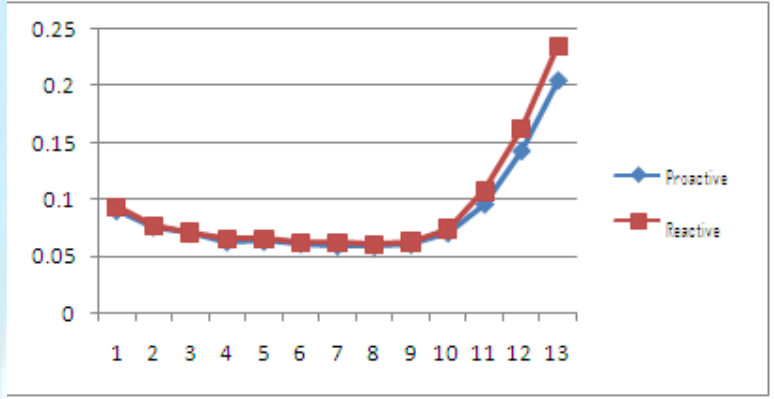

\section{Normalized Routing Load Comparison}

The normalized routing overhead of the proactive approach remains almost constant for a particular advertisement interval irrespective of the pause time. Whereas in case of reactive approach with decreasing pause time, the gateway discoveries need to be invoked more often due to frequent link breaks. Moreover, as the reactive approach continues using longer and older routes and does not use route optimization until the route is broken, the chances of link breaks also increases. This further adds to the number of route discoveries. With this greater number of gateway discoveries, the control traffic also increases, which ultimately results in higher normalized routing load.

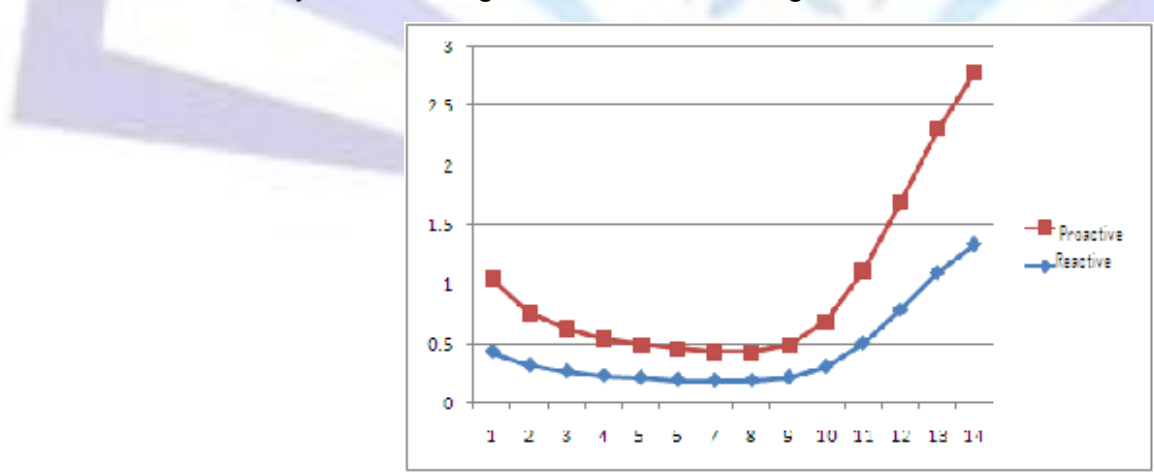

\section{CONCLUSIONS}

In this paper gateway call the MRA message for all gateway discovery schemas in MANETs. Basically, The node which is unstable but have route to gateway are prone to receive the MRA messages while in stable zones the overhead originated by MRA messages are avoided. The evaluation of the algorithm shows the goodness of the proposal. The simulations were performed with various types of movements including synthetic and realistic mobility models.

In the paper, MANET routing protocol-AODV has been extended to route packets, not only within a MANET but also between a wireless MANET and the wired network. The communication between the wireless and the wired network must pass through these nodes, which are referred to as gateways. In this paper, three methods for detection of these 
gateways have been presented, implemented and compared. The three methods for gateway detection are referred to as reactive, proactive and hybrid gateway discovery. The comparison between these methods provides us useful information.

Regarding the packet delivery ratio in proactive gateway discovery approach, due to regular exchange of gateway information, routes are always optimized and the nodes have fresher and shorter routes to the destination. This reduces the chances of link breaks and increases the packet delivery ratio. On the other hand in reactive approach, a node continues to use a longer route until it is broken even if an alternate shorter route is available. This reduces the packet delivery fraction. In terms of the average end-to -end delay, the reactive gateway discovery suffers from less average endto-end delays compared to proactive discovery approach.

\section{REFERENCES}

[1] Bhargavan, V., Das, B.: Routing in Ad Hoc Networks Using Minimum Connected Dominating Sets. International Conference on Communications'97, Montreal, Canada, June. (1997)

[2] Lin, C. R., Gerla, M.: Adaptive clustering for mobile wireless networks. IEEE Journal on Selected Areas in Communication. (1996) 1265-1275

[3] Yi, Y., Gerla, M., Kwon, T. J.: Efficient flooding in Ad Hoc Networks using On-Demand (Passive) Cluster Formation. Proc. of MobiHoc. (2002)

[4] Johnson, D. B., Maltz, D. A.: Dynamic source routing in Ad Hoc Networks. Mobile Computing. (2000) 153-181

[5] Perkins, C., Royer, E., Das, S.: Ad hoc on Demand Distance Vector (AODV) Routing. Internet draft, IETF, October. (1999)

[6] ] R. Fallier and B. Scheers, "Clustering in Mobile Adhoc Networks".

[7] K. Fall \& K. Varadhan. (2007). "The ns Manual (formerly ns Notes and Documentation). TheVINT Project".

[8] M.Geetha, Dr. R. Umarani, "Performance Comparison and Analysis of AODV and DSDV Gateway Discovery Protocol in MANET", International Journal of Engineering Science and Technology, Vol. 2(11), 2010, 6521-6531

[9] Belding-Royer E.M., Sun Y., Perkins C.,"Global Connectivity for IPv4 Mobile Ad-Hoc Networks", IETF Internet Draft, Nov. 2001. Work in progress.

[10] P. Ratanchandani and R. Kravets, "A Hybrid Approach to Internet Connectivity for Mobile Ad hoc Networks", in Proc. of the IEEE WCNC 2003, Vol. 3, pp. 1522-1527. New Orleans, USA, March 2003.

[11] Jaewook Shin, Haeryong Lee, Jeehyeon Na, Aesoon Park and Sangha Kim" Gateway Discovery and Routing in Ad Hoc Networks withNAT-based Internet Connectivity" 0-7803-8521-7/04/\$20.00 @ 2004 IEEE.

[12] P. M. Ruiz and A. F. Gomez-Skarmeta, "Enhanced Internet Connectivity for Hybrid Ad Hoc Networks Through Adaptive Gateway Discovery", in Proc. of the 29th IEEE International Conference on Local Computer Networks (LCN'04), Tampa, Florida, November 2004.

[13] P. M. Ruiz and A. F. Gomez Skarmeta, "Maximal Source Coverage Adaptive Gateway Discovery for Hybrid Ad Hoc Networks", Lecture Notes in Computer Science, vol.3158, July 2004, pp.28-41.

[14] P. M. Ruiz and A. F. Gomez-Skarmeta, "Adaptive Gateway Discovery Mechanisms to Enhance Internet Connectivity for Mobile Ad Hoc Networks", Ad Hoc \& Sensor Wireless Networks, Vol. 1, 2005, pp. 159-177.

[15] Khaleel Ur Rahman Khan, Ehthesham M., Kumar M., Rafi UZaman, "An Effective Gateway Discovery Mechanism in an Integrated Internet-MANET (IIM)", IEEE, 2008

[16] H. Ammari and H. El-Rewini, " Integration of Mobile Ad Hoc Networks and the Internet using Mobile Gateways,"Proceeding of the 4th International Workshop on Algorithms for Wireless, Mobile, Ad Hoc and Sensor network.

[17] Kumar Rakesh,Anil Kumar Surje and Manoj Mishra "Review Strategies And Analysis of Mobile Ad hoc Networks Internet Integration Solutions" IJCSI International Journal of Computer Science Issues, Vol. 7, Issue 4, No 6, July 2010.

[18] Koushik Majumder, Dr. Sudhabindu Ray, Prof. Subir Kar Sarkar, "Implementation and Performance Evaluation of the Gateway Discovery Approaches in the Integrated MANET Internet Scenerio", International Journal on Computer Science and Engineering, Vol.3 No.3, March 2011, PP: 1213-1226.

[19] Kumar R. "Performance Evaluation of Gateway Discovery Approaches in the Integrated Mobile Ad Hoc Network (MANET)-Internet Scenario" International Journal of Computer Technology and Electronics Engineering (IJCTEE) Volume 2, Issue 3, June 2012.

[20] Belding-Royer E.M., Sun Y., Perkins C.,"Global Connectivity for IPv4 Mobile Ad-Hoc Draft, Nov. 2001. Work in progress.

[21] J. Yoon, M. Liu \& B. Noble.(2003). "Random waypoint considered harmful." In Twenty-Second Annual Joint Conference of the IEEE Computer and Communications Societies. 
[22] P. M. Ruiz and A. F. Gomez-Skarmeta, "Enhanced Internet Connectivity for Hybrid Ad Hoc Networks Through Adaptive Gateway Discovery", in Proc. of the 29th IEEE International Conference on Local Computer Networks (LCN'04), Tampa, Florida, November 2004.

[23] S. Bin, S. Bingxin, L. Bo, H. Zhonggong, and Z. Li, "Adaptive gateway Discovery Scheme for Connecting Mobile Ad Hoc Networks to the Internet," Proceedings of International Conference on Wireless Communications, Networking and Mobile Computing, vol. 2, pp. 795-799, 2005

[24] A. Hamidian, U. Korner, A. Nilsson, "Performance of internet access solution in mobile ad hoc networks", Lecture Notes in Computer Science 3427 (1) (2005) 189-201.

[25] Kumar Rakesh, and Misra, M. "An Efficient Mechanism for Connecting MANET and Internet through Complete Adaptive Gateway Discovery", In Proceedings of the First International Conference on Communication System Software and Middleware (COMSWARE2006). New Delhi, India, January 2006, 1-5.

[26] Yuste, A.J., Trujillo, F.D., Trivĩno, A., Casilari, E.: "An adaptive gateway discovery for mobile ad hoc networks". In: 5th ACM international workshop on Mobility management and wireless access (MOBIWAC), Chania, Crete Island, Greece, October 2007, pp. 159-162 (2007).

[27] Yuste, A.J., Trujillo, F.D., Trivĩno, A. and Casilari, E" Connectivity Gateway Discovery in MANETs" Springer-Verlag Berlin Heidelberg L. Cerd`a-Alabern (Ed.): Wireless and Mobility , LNCS 5122, pp. 128-141, 2008

\section{Author's biography}

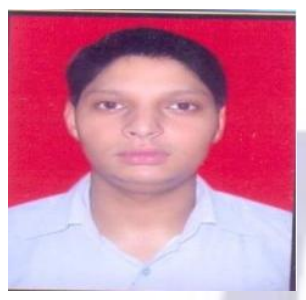

Mahesh kumar Tiwari is a student of M.Tech in Department of Computer Science \& Engineering in IIMT Engineering College, Meerut affiliated to Uttar Pradesh Technical University, Lucknow, Uttar Pradesh \& B.Tech degree in Computer Science \& Engineering from Bharat Institute of Engineering and Technology, Meerut, affiliated to Uttar Pradesh Technical University, Lucknow, Uttar Pradesh.

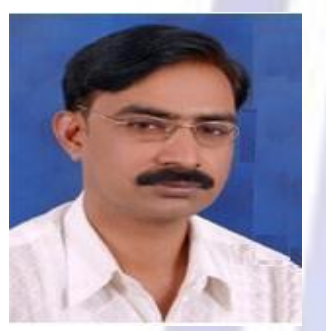

The author received his M.Tech degree in Computer Science \& Engineering from IIMT Engineering College, Meerut affiliated to Uttar Pradesh Technical University, Lucknow, Uttar Pradesh \& B.Tech degree in Computer Science \& Engineering from Bundelkhand Institute of Engineering and Technology, Jhansi, now affiliated to Uttar Pradesh Technical University, Lucknow, Uttar Pradesh. Presently he is the Ph.D research scholar in IFTM state University. He has 17 years of teaching experience in Technical education. He is Ex-faculty of Kumaon Engineering College Dwarahat, Almora (Uttarakhand), a Govt. Institute and presently working in IIMT Engineering College Meerut (U.P) as Faculty \& M.Tech Co-ordinator of Computer Science \& Engineering and Information Technology department. He has published a number of research papers in the field of Ad-Hoc Networks and internet access solution for MANETs. His research area is Ad hod Networks, protocol design for MANETs, Internet access solutions for MANETs etc. He is holding the membership of different professional technical bodies like IACSIT, IAENGG and IJMIA etc.

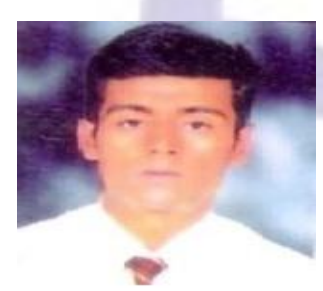

The author received his M.Tech degree in Computer Science \& Engineering from IIMT Engineering College, Meerut affiliated to Uttar Pradesh Technical University, Lucknow, Uttar Pradesh \& B.Tech degree in Computer Science \& Engineering from Meerut Institute of Engineering and Technology, Meerut, affiliated to Uttar Pradesh Technical University, Lucknow, Uttar Pradesh. He has 5 years of teaching experience in Technical education. He is presently working in IIMT Engineering College Meerut (U.P) as Faculty \& His research area is Ad hod Networks, protocol design for MANETs, Internet access solutions for MANETs etc. 